\title{
Applications of Protein Engineering to Members of the Old Yellow Enzyme Family
}

\author{
Erica D. Amato and Jon D. Stewart* \\ Department of Chemistry, 126 Sisler Hall, University of Florida, Gainesville, FL \\ 32611 USA
}

Phone \& Fax 352.846.0743, E-mail jds2@ @ chem.ufl.edu

*Author to whom correspondence should be addressed 


\begin{abstract}
In the 20 years since Massey's initial report in 1995, interest in using alkene reductases to prepare chiral intermediates for synthesis has grown rapidly. While native alkene reductases often show very high stereoselectivities toward favorable substrates, these enzymes have somewhat size-restricted active sites that limit their substrate ranges to small alkenes. In addition, most alkene reductases have the same stereoselectivities, which makes it difficult to access the "other" product enantiomers. Protein engineering strategies have been used to address both of these issues and good progress has been made in several cases. This review summarizes published examples through late 2014 and focuses on studies of six enzymes: Saccharomyces pastorianus OYE 1, tomato OPR1, Zymomonas mobilis NCR, Enterobacter cloacae PB2 PETN reductase, Bacillus subtilis YqjM and Pichia stipitis OYE 2.6.
\end{abstract}




\section{Introduction}

Enzyme-catalyzed alkene reductions have grown significantly in popularity since the original report by Massey in $1995 .{ }^{1}$ Despite being the first-discovered flavoprotein, ${ }^{2}$ the physiological role(s) of most old yellow enzyme (OYE) family members remain obscure, even to this day. Nonetheless, these biocatalysts have been used to reduce a large number of electron-deficient alkenes, often with very high stereoselectivities (for recent reviews, see 3-5 and references therein). In addition to defining the synthetic utility of OYEs, these first-generation studies with wild-type enzymes pointed out two key deficiencies.

First, the relatively small active sites of most OYEs limit them to correspondingly small substrates. This is not a serious problem so long as OYEs are employed early in a synthetic sequence; however, more advanced intermediates are typically larger. This means that chirality must be introduced early and carried through the majority of the sequence. This provides multiple opportunities for unwanted racemization and / or epimerization. For this reason, it would be more desirable to synthesize a late-stage prochiral alkene, then reduce it with high stereoselectivity at or near the end of the synthetic sequence.

The second problem with OYEs is that their stereoselectivities are nearly always the same. ${ }^{6}$ For synthetic applications, pairs of enantiocomplementary alkene reductases would be ideal. Most efforts to identify OYEs with reversed stereoselectivity by cloning and overexpressing homologs have met with disappointment. One partial solution involves changing the configuration of the starting alkene: at least in some cases, a single OYE can provide both product antipodes when presented with $(E)$ - and $(Z)$-starting materials. Unfortunately, altering the alkene geometry is not always straightforward, and is impossible in cyclic alkenes. ${ }^{7}$ 
Since wild-type OYEs are usually lacking in one or more desirable traits for synthetic applications, protein engineering is the logical choice for overcoming these deficiencies. While the mutagenesis strategies have ranged from highly random to highly rational, several have successfully yielded variants with improved properties. These studies are the focus of the present review, which is organized by enzyme. Earlier reviews in this area should be consulted for discussions of OYE substrate range and stereoselectivity.

\section{Saccharomyces pastorianus OYE 1}

S. pastorianus ${ }^{8}$ OYE 1 was first reported in 1932 and it has been the best-studied member of the OYE family. Much of our knowledge of the OYE family in general derives from the extensive studies of S. pastorianus OYE 1 carried out in the Massey lab. In addition, the x-ray crystal structure was solved by Fox and Karplus, ${ }^{9,10}$ which has enabled sophisticated structurefunction studies. One key early observation was that the tightly bound FMN cofactor was reduced by NADPH, leaving NADP ${ }^{+}$to dissociate from the active site. The reduced FMN could be reoxidized by electron transfer to a variety of non-physiological acceptors, e.g., ferricyanide.

Oxygen could also fulfill the electron acceptor role (forming hydrogen peroxide), although the slow rate for its reduction suggested that it was not the native partner. In fact, the physiological electron transfer partner of OYE 1 remains obscure; a report that it protected the cytoskeleton of yeast from oxidative stress by reducing a disulfide bond in actin ${ }^{11}$ was later shown to be erroneous. ${ }^{12}$

The field of enzyme-catalyzed alkene reductions grew rapidly after the first report by Massey that S. pastorianus OYE 1 could transfer electrons from reduced FMN to a variety of electrondeficient alkenes. ${ }^{1}$ Alkenes conjugated to aldehydes, ketones and nitro groups were the best substrates, which nicely complemented the substrate range of transition metal-catalyzed 
hydrogenations. Deuterium labeling revealed a net trans-addition of $\mathrm{H}_{2}$ across the olefin, a consequence of hydride addition to one face while the proton is donated by the side-chain of Tyr 196 from the opposite side (Scheme 1). Two key hydrogen bonds are formed between the sidechains of His 191 and Asn 194 and the substrate carbonyl. These position the substrate in the active site and also electronically activate the $\pi$ system for hydride addition at the $\beta$-carbon. A crystal structure with an NADPH analog suggests that these hydrogen bonds also interact with the carboxamide side-chain of the nicotinamide, properly positioning $C_{4}$ for hydride transfer to FMN in the reductive half-reaction. ${ }^{13}$

The numerous enzyme-substrate interactions explain why OYE 1 shows very high stereoselectivities in alkene reductions. That these residues are highly conserved in other OYE 1 homologs also explains why these enzymes nearly always show the same stereoselectivities as OYE 1. Finally, the relatively modest size of the active site pocket restricts OYE 1 to smaller substrates, particularly at the $\beta$-position of enones. ${ }^{14}$ Protein engineering studies of $S$. pastorianus OYE 1 have therefore generally focused on changing its stereoselectivity and / or expanding its substrate range. In early work, a number of site-directed mutants were created in S. pastorianus OYE 1 to probe the roles of several residues in the catalytic mechanism; ${ }^{15-20}$ mutations designed to improve its traits for chemical synthesis are summarized below.

Altering the stereoselectivity of S. pastorianus OYE 1. The malleability of OYE 1 stereoselectivity was a chance observation. In an effort to increase the active site volume (to accommodate larger $\beta$-substituted cyclohexenones), site-saturation mutagenesis was applied to Trp 116. While catalytic activity toward larger substrates was improved modestly in some variants, the Trp 116 Ile mutant reduced $(S)$-carvone with reversed stereoselectivity as compared to the wild-type (98\% conversion and $88 \%$ de). ${ }^{21}$ This was a completely unexpected result and it 
was the first indication that OYE stereoselectivity was not immutable. Deuterium labeling was consistent with the notion that $(S)$-carvone reduction by the Trp 116 Ile mutant followed the same mechanism (net trans-addition of $\mathrm{H}_{2}$ across the alkene) and it was speculated that the substrate bound in a reversed orientation that placed the opposite face of the $\pi$ system parallel to the FMN. Computer docking studies were generally consistent with this hypothesis. ${ }^{21}$

These promising results prompted a systematic study of Trp 116 variants and their stereoselectivities. $^{22,23}$ This work had two goals. First, it was hoped that additional OYE 1 variants would enlarge the range of substrates showing reversed stereoselectivity beyond only $(S)$-carvone. The second goal was to understand - at the molecular level - why replacing one hydrophobic side-chain (Trp) with another (Ile) at a single position had such significant impacts. The complete collection of Trp 116 variants was therefore overexpressed and tested against both $(S)$ - and $(R)$-carvone. The mutants fell into four categories: those with reversed stereoselectivity for both $(S)$ - and $(R)$-carvone (Ala and Val), those with reversed enantioselectivity for (S)-, but normal for $(R)$-carvone (Asn, Gln, Ile and Met), those with unchanged stereoselectivity toward either carvone enantiomer (Asp, His, Leu, Phe, Pro, Tyr and Trp) and those that had lost catalytic activity (Arg, Cys, Gly, Glu, Lys, Ser and Thr). The remarkable observation was that Val, Leu and Ile - normally considered nearly equivalent - fell into three different categories of stereoselectivity. The results also underscore how fine details of protein architecture dictate changes in enantioselectivity. For this reason, it was essential to obtain detailed structural information on the OYE 1 mutants, preferably in complex with substrates, in order to understand the impact of Trp 116 mutations.

The conditions developed by Fox and Karplus readily yield OYE 1 crystals that diffract to very high resolutions (typically $1.2-1.6 \AA$ A). ${ }^{9}$ This, along with the efficient affinity 
purification method developed by Massey, ${ }^{24}$ made it possible to analyze a large number of Trp 116 variants of OYE 1 with altered stereoselectivities, both in free form and complexed with substrates. The latter forms are pseudo-Michaelis complexes that cannot proceed further because the FMN remains in the oxidized form; ${ }^{25}$ nonetheless, they provided very valuable insights. The two alternate carvone binding modes ("normal" and "flipped"; Scheme 2) place the isopropenyl side-chain into one of two active site pockets and both arrangements were observable by x-ray crystallography in the appropriate mutants. Placing the side-chain in the location needed for the "flipped" orientation, however, is blocked by the indole side-chain of Trp 116 in wild-type OYE 1 (Figure 1). For this reason, the side-chain must lie in the only available pocket, despite its somewhat hydrophilic character. This corresponds to the "normal" binding mode. When Trp 116 is exchanged for a smaller residue, both active site pockets are accessible. In this situation, both carvone enantiomers strongly prefer to bind in the "flipped" orientation, perhaps because their hydrophobic isopropenyl moieties project into the newly-accessible cavity, which is lined by hydrophobic side-chains. Seen in this light, it is actually wild-type OYE 1 whose stereoselectivity is unusual: when the active site is opened, a hydrophobic substrate strongly prefers to bind in the "flipped" orientation, even though the "normal" orientation still remains sterically accessible.

S. pastorianus OYE 1 Trp 116 variants were also used to reduce a set of three BaylisHillman adducts (Scheme 3). ${ }^{23}$ Wild-type OYE 1 performed rather poorly (<50\% conversion); however, several mutants gave good $(S)$-selectivities and high conversions. The Trp 116 Ile mutant performed well (98\% conversion), and its structure was therefore solved after soaking crystals with a cyclopentenone substrate. An arrangement consistent with hydrogen bonding between the hydroxyl group of the substrate side-chain and the side-chain of Thr 37 was 
observed. This interaction would be sterically impossible in the wild-type enzyme.

Interestingly, substrate binding in the catalytically productive, "flipped" orientation re-orients the side-chain of the Ile at position 116, which in turn shifts the side-chain of His 191, which then alters the placement of Tyr 196. The latter residues form an essential hydrogen bond with the substrate carbonyl and act as the general acid, respectively (Figure 2). The changes provoked by substrate binding are consistent with an induced fit model in which OYE 1's catalytic machinery is only assembled into the required architecture by a correctly fitting substrate.

While changes for Trp 116 slightly increased the active site volume of S. pastorianus OYE 1, the alterations did not significantly enlarge the acceptable substrate size. The circular permutation studies of Lutz have dramatically improved this aspect of S. pastorianus OYE 1 along with its catalytic efficiency. ${ }^{26}$ OYE 1 contains approximately 400 amino acids, and clever molecular biology provided a set of circularly-permuted variants in which the original $\mathrm{N}$ - and $\mathrm{C}$ termini of the protein were joined by a three amino acid linker (Gly-Thr-Ser) and new termini were introduced by breaking the chain at a different position. This was done in parallel after nearly every even-numbered amino acid to yield a collection of almost 200 variants. ${ }^{27}$ Additional members were added from odd-numbered positions after an initial round of screening had identified influential regions within the sequence.

The library of OYE 1 variants was produced individually by an in vitro transcription / translation strategy well-suited to parallel studies, and proteins were screened against ketoisophorone to identify variants with catalytic activities equal to or greater than the wild-type. These clustered into four regions of the polypeptide chain. The rate of the best variant was 19fold higher than the starting protein and all the circularly-permuted variants shared the wild-type $(R)$-stereoselectivity. Screening was extended to two additional substrates (trans- 
cinnamaldehyde and $(S)$-carvone); the same four regions of the OYE 1 sequence provided the best rate improvements for these substrates as well. Pre-steady state kinetics suggested that the improved catalytic efficiencies were largely due to an increased rate of $\mathrm{NADP}^{+}$dissociation at the conclusion of the reductive half-reaction. ${ }^{26}$ This step is rate-limiting in wild-type OYE 1, and the altered structural dynamics and changes in active site volume caused by moving the polypeptide termini to new locations is consistent with this explanation.

\section{Lycopersicon esculentum (tomato) 12-oxophytodienoate reductase 1 (OPR1)}

OPR1 is one of three homologous OYEs found in the tomato genome and it shares high sequence similarity with S. pastorianus OYE 1 . Some members of the plant OPR family catalyze an essential step in the jasmonic acid biosynthetic pathway. ${ }^{28-33}$ While the native substrates are 2-cyclopentenones, OPRs also accept a variety of electron-deficient alkenes, which has made them useful biocatalysts for synthesis. ${ }^{34,35}$

Recently, tomato OPR1 was used to prepare a key intermediate in the synthesis of $(S)$ Pregabalin (Scheme 4). ${ }^{36}$ This was part of a larger study of $\beta$-cyanoacrylate ester reductions by several OYE family members by the Faber and Wong groups. As noted previously, (E)- and (Z)substrates were sometimes reduced to opposite enantiomers ( $S$ - and $R$-, respectively, in this case), although rates generally declined significantly as the size of the $\beta$-substituent increased. This was particularly troublesome because the Pregabalin precursor has a sterically demanding $\beta$-isobutyl group. Methyl esters were generally better substrates than their ethyl counterparts, an opportunity for "substrate mutagenesis" that is often underappreciated. Unfortunately for $(S)$ Pregabalin synthesis, none of the wild-type enzymes provided acceptable conversions and stereoselectivities. 
Rational mutagenesis was applied to tomato OPR1 to obtain a more practically useful biocatalyst for this application. ${ }^{26}$ Mutations were chosen by sequence comparisons to other OYE family members and the best changes were Cys 20 Tyr, Ile 287 His and His 245 Asp. All three variants provided the $(S)$-product in $>99 \%$ ee, although conversions remained below $20 \%$.

\section{Zymomonas mobilis nicotinamide-dependent cyclohexenone reductase (NCR)}

Rosche et al. discovered a very active alkene reductase of the OYE family in the bacterium Z. mobilis that was designated $\mathrm{NCR} .{ }^{37}$ After its initial isolation, NCR was tested against a panel of representative alkenes, where it showed very good performance, particularly for the geometric isomers present in citral ( $>93 \%$ conversion and $>95 \%$ ee). ${ }^{38}$

The Pietruszka group used Z. mobilis NCR to test the ability of sequence-based enantioselectivity predictions. ${ }^{39}$ It had been suggested that OYE stereoselectivity depended critically on the nature of the amino acid at a position in loop $\beta 2 .^{40}$ Site-saturation mutagenesis was applied to the corresponding position in Z. mobilis NCR (amino acid 67). Wild-type NCR shows $(S)$-stereoselectivity toward typical substrates; all six mutants predicted to yield the opposite enantiomers actually retained the wild-type stereopreference. The authors conclude that stereoselectivity in OYEs is more broadly determined, although it should be noted a single amino acid change may also be correlated with other sequence alterations that together have larger impacts.

The Hauer group recently applied rational mutagenesis of loops in Z. mobilis NCR in an effort to improve its stability under process conditions. ${ }^{41}$ This enzyme is a member of the "classical" OYE family, which differ from the "thermophilic-like" subgroup that includes Bacillus subtilis YqjM (vide infra). Members of the latter subgroup have shorter surface loops, and it was hypothesized that the thermal- and solvent stability of a "classical" OYE could be 
increased by borrowing this feature. Specifically, loop 3 was shortened by 7 amino acids and 4 amino acids were removed from loop $4 .{ }^{42}$ The two loop deletion variants generally had modestly lower catalytic activities at room temperature than the wild-type NCR and the denaturation temperatures were almost unchanged. On the other hand, when an alkene reduction was conducted at elevated temperatures, the shortened loop 4 variant out-performed wild-type NCR and it also showed higher resistance to organic solvents such as acetone, $i$-PrOH, EtOAc and THF. By contrast, the shortened loop 3 variant was more susceptible to solvent-induced and temperature-mediated inactivation.

Several protein engineering strategies have been used to influence the catalytic efficiency and enantioselectivity of $Z$. mobilis NCR. The crystal structure was determined (PDB code 4A3U), and this information was used to guide rational mutagenesis. ${ }^{43}$ In one approach, sequence comparisons between "classical" and "thermophilic" OYE subgroups were used to identify residues that were conserved within each subgroup, but differed between the subgroups. This analysis yielded eight single amino acid changes that were introduced into the NCR sequence individually. Each was tested against a panel of five substrates that included both cyclic and acyclic structures to determine the effect on conversion efficiency and stereoselectivity. Unfortunately, none of the mutants had significantly better catalytic efficiencies or different enantioselectivities as compared to the wild-type and several mutants were nearly inactive. On the other hand, when reactions were scaled up, two variants (Pro 245 Gln and Asp 337 Tyr) gave higher rates during the first two hours, although this value declined to match that of the wild-type afterward.

Loop grafting was also employed in an effort to improve NCR. ${ }^{43}$ Regions targeted for replacement were found by multiple sequence alignments, again focusing on regions of the 
protein that were conserved within sub-families, but differed between them. This analysis also used the crystal structure of both OYE 1 and NCR to determine the best locations to begin and end each loop grafting site. Four loop graft mutants of NCR resulted from this analysis in which loops 1 - 4 were replaced in NCR with their OYE 1 counterparts. Unfortunately, while one loop graft mutant was active, albeit impaired (57\% conversion 93\% ee), the other three variants showed almost no conversion for any of the five substrates tested.

\section{Enterobacter cloacae PB2 Pentaerythritol Tetranitrate (PETN) reductase}

PETN reductase is an OYE family member that is part of a metabolic pathway that allows $E$. cloacae PB2 to use explosives such as pentaerythritol tetranitrate (PETN) and glycerol trinitrate as its sole nitrogen source. ${ }^{43}$ The crystal structure of PETN reductase was reported by Barna et al. in 2001 (PDB code 1H50); ${ }^{44}$ a region of unexplained electron density between the active site ligand (picric acid) and the side-chain of Trp 102 was later shown to reflect multiple conformations of the Trp 102 indole, rather than a protein-ligand covalent bond. ${ }^{45}$ The Scrutton group has carried out extensive studies with PETN reductase, showing its utility in reducing a variety of electron-deficient alkenes with very high stereoselectivities. ${ }^{46,47}$ Very recently, this group reported the unexpected influence of oxygen on the stereoselectivities of PETN reductasecatalyzed nitroalkenes reductions. ${ }^{48}$

PETN reductase has been well-characterized, both with respect to structure and substrate range. Moreover, because this enzyme can reduce some explosives and thereby detoxify them, it has been subjected to significant protein engineering efforts. The Scrutton group developed an automated, high-throughput workflow that encompassed colony picking, cell growth, PETN reductase purification and steady state rate measurements under anaerobic conditions. ${ }^{49,50}$ Several residues were targeted for site-saturation mutagenesis; they were chosen because they 
were known or predicted to be involved in substrate binding (His 181, His 184, Tyr 351), in catalysis (Tyr 186, the putative proton donor) or because they were close to the active site (Thr 26, Tyr 68, Trp 102 and Gln 241). The best rate improvements were observed when Trp 102 was replaced by Phe and Ile, which provided 5- and 6-fold higher rates, respectively, against a sterically demanding enone substrate. Interestingly, Trp 102 of PETN reductase corresponds to Trp 116 of OYE 1, further underscoring the general importance of this residue in OYE family members. The Thr 26 Ala (0.8 fold compared to w.t.) and Gln 241 Trp (0.9 fold compared to w.t.) mutants also showed improved catalytic activities, albeit with lower rates. Finally, both the Ile and Phe replacements for Trp 102 had improved catalytic efficiencies against a subset of the substrates tested. Of all the mutants, two were most interesting. Trp 102 Phe reduced a nitroalkene at a faster rate than the wildtype and also had an inverted enantiopreference. The Thr 26 Ser variant also exhibited reversed enantioselectivity toward a nitroolefin. Key variants were isolated on larger scales for more extensive characterization.

The crystal structure of the Thr 26 Ser mutant was solved to $1.5 \AA$ (PDB code 3P67) in order to understand its altered stereopreferences. ${ }^{50}$ In addition, the authors solved the structure of $\mathrm{His}_{8^{-}}$ tagged wild-type PETN reductase to $1.4 \AA$ to ensure that the $C$-terminal affinity tag did not change the structure (PDB code 1H50). ${ }^{51}$ The active site of the Thr 26 Ser mutant was nearly the same as the wild-type, although the position of Tyr 351 changed slightly. The highly-conserved hydrogen bond between the residue at position 26 and the FMN $O_{4}$ atom was retained in the mutant.

Because of its central location in the PETN reductase active site, Tyr 351 was targeted for site-directed mutagenesis. ${ }^{52}$ Crystal structures were solved for the Ala, Phe and Ser variants (PDB codes 3P84, 3P81, and 3P8J). In general, the Tyr 351 mutants showed decreased catalytic 
activities, although one reaction showed increased enantioselectivity. Their largest impact was to eliminate formation of an oxime product (formed by nitro, rather than alkene, reduction). Selected Tyr 351 mutations were combined with the previously-discovered Thr 26 Ser alteration to identify possible synergistic effects. ${ }^{53}$ Unfortunately, this was generally not observed, at least in the cases that were probed.

A second study by the Scrutton group focused on changes at His 181 and His $184 .^{53}$ In the "classical" OYE mechanism, these residues form essential hydrogen bonds with the carbonyl oxygen of enone and enal substrates (for example, see 15). For nitroolefins, the roles and importance of these imidazole side-chains is less clear. A nearly complete set of amino acid replacements for both His 181 and His 184 was prepared and their catalytic properties evaluated after purification. Reactions were conducted anaerobically and multiple substrates were assessed including an enone, enal and several nitroolefins. Changes to His 181 severely impaired catalytic activity; altering His 184 was better tolerated. Several mutants showed significantly better stereoselectivities and the best was the His 184 Asn variant ( $85 \%$ ee and 95\% de). In several cases, enhanced oxime production from a nitroolefin was observed. Crystal structures of the His 181 Asn and His 184 Asn mutants were solved to determine whether the enhanced oxime production could be explained by the structural changes observed (PDB codes 3P74 and 3P82). ${ }^{54}$

\section{Bacillus subtilis YqjM}

The Reetz group pioneered the strategies of Combinatorial Active site Saturation Testing (CASTing) and iterative saturation mutagenesis (ISM) to improve enzyme properties (for recent reviews, see ${ }^{54,55}$ and references therein). A 2009 study by Bougioukou et al. applied these methodologies to a bacterial OYE (Bacillus subtilis YqjM). ${ }^{56}$ The reduction of $\beta$-methyl-2- 
cyclohexenone was chosen as the model reaction. Wild-type YqjM reduces this substrate with poor conversion $(\mathrm{ca} .3 \%)$ and $76 \%$ ee $(R)$.

Starting from the X-ray crystal structure with bound inhibitor (PDB code 1Z42) ( $p$ hydroxybenzoate), twenty residues were chosen for site-saturation mutagenesis, based on their proximity to the catalytic site. Three nearby residues were excluded from the list of targets since they form critical hydrogen bonds to the substrate carbonyl (His 164 and His 167) or serve as the general acid for enol(ate) protonation (Tyr 169). One important aspect this study was an emphasis on library quality, i.e., ensuring that the anticipated sequence diversity is actually present in the clones that are screened. Because wild-type YqjM has almost no catalytic activity toward the enone substrate, groups of 8 mutants were pooled prior to GC analysis, which significantly reduced the screening burden. Nine of the 20 libraries contained "hits" and both $(R)$ - and $(S)$-selective mutants were identified. The best variants were used as "anchor sequences" that were randomized at other positions that had been shown to influence catalysis. The most (R)-selective mutant (Cys 26 Asp / Ile 69 Thr; 94\% ee) also showed a 130-fold improvement in catalytic rate. Interestingly, $(S)$-selective mutants were not always highly efficient catalysts. The best combination (Cys 26 Gly / Ala 60 Val) gave $>99 \%$ ee and $\mathrm{ca}$. $80 \%$ conversion. That only a few rounds of ISM were required to improve catalytic activity and stereoselectivity simultaneously testifies to the power of the methodology.

The best variants from the ISM program were then tested against a variety of other substrates to determine the generality of the improvements. ${ }^{56}$ This was indeed the case.

\section{Pichia stipitis OYE 2.6}

The most recent protein engineering effort to alter alkene reductase stereoselectivity was based on Pichia stipitis OYE 2.6. ${ }^{57}$ This enzyme was originally annotated as a putative 
oxidoreductase from a genome sequencing project of a xylose-fermenting yeast. ${ }^{58}$ What makes it unusual is that, unlike S. pastorianus OYE 1 and $c a .90 \%$ of its homologs, the native sequence of OYE 2.6 contains Ile at the site of the highly conserved active site Trp. ${ }^{59}$ In addition to Ile in place of Trp, OYE 2.6 also differs from $S$. pastorianus OYE 1 in the length of two active site loops, including loop 6, which must move in order to bind and release $\mathrm{NADP}(\mathrm{H})$. Wild-type OYE 2.6 is also more stable than S. pastorianus OYE 1 and shows complementary stereoselectivity in some cases. ${ }^{60}$ It was therefore selected as a platform to evolve variants with complementary enantioselectivities.

The crystal structure of $P$. stipitis OYE $2.6^{61}$ was analyzed (PDB code 3TJL) and 13 residues whose side-chains lay near the center of the active site were selected for site-saturation mutagenesis. Three Baylis-Hillman adducts were chosen as test substrates (Scheme 3). Wildtype $P$. stipitis OYE 2.6 reduces all three alkenes to the corresponding $(S)$-products with very high enantioselectivities. ${ }^{23}$ Beyond the fact that reduction products $\mathbf{2 , 4}$ and $\mathbf{6}$ are useful chiral intermediates, this study also probed the question of whether a general solution to evolving enantiocomplementarity might exist, or whether each substrate would require its own pathway through sequence space. The molecular biology methods were optimized to ensure that all libraries were sufficiently diverse and contained all of the targeted amino acid replacements. ${ }^{62}$

For cyclopentenone $\mathbf{5}$, the pathway from $(S)$-selectivity (wild-type) to $(R)$-selectivity was straightforward. The pivotal change was Tyr 78 Trp, which provided 63\% ee $(R)$ on its own. The crystal structure of this variant showed that Trp substitution blocked the position of an ordered water molecule found in the wild-type structure. This ordered solvent likely corresponds to the position of the side-chain hydroxyl of the substrate, and this forces substrate to bind in a "flipped" orientation that eliminates steric clash. ${ }^{63}$ A second-generation library focused on 
position 113 improved $(R)$-stereoselectivity even further. No additional efforts were made for this substrate since the enantioselectivity target had been reached after two rounds.

Switching enantioselectivity for acyclic Roche ester precursor 1 required a more complex strategy. None of the 13 primary site-saturation mutagenesis libraries yielded any $(R)$-selective variants. Moreover, the pivotal mutant observed previously (Tyr 78 Trp) showed essentially no catalytic activity toward this substrate. Despite these apparently bleak prospects, second-round libraries were centered on variants that were successful in altering the enzyme's stereoselectivity toward one of the other two substrates in the study. This was successful when the Tyr $78 \operatorname{Trp}$ mutant was additionally randomized at position 113: the Cys, Leu, Phe and Val mutants not only regained catalytic activity toward the substrate, but also provided the $(R)$-product in ca. $90 \%$ ee. ${ }^{57}$ The crystal structure of the Tyr 78 Trp / Ile 113 Cys mutant (PDB code 4M5P) suggested a logical reason for these observations. The Tyr 78 Trp mutant (PDB code 4QAI) precluded substrate binding in the "normal" orientation as described above; however, in this case, the "flipped" orientation was also unacceptable because the methyl ester would lie too close to the side-chain of Ile 113. Because neither binding mode was accessible, the net result was no catalytic activity toward this substrate. $(R)$-Stereoselectivity was improved even further by altering a residue on the opposite side of the active site (Phe 247).

In addition to providing a synthetically useful biocatalyst that yields $(R)$-Roche ester, this study also showed how one can cross a "desert" of sequence space. ${ }^{64}$ There is no direct path from the wild-type to the final triple mutants in which all the intermediate protein variants retain catalytic activity. In an ordinary directed evolution project, this cannot be overcome and the effort must be abandoned. On the other hand, by carrying out three parallel directed evolution 
projects, one can use comparative data to leap over the "desert" and land in a productive island of sequence space.

Inverting the stereoselectivity of P. stipitis OYE 2.6 for the final substrate in this study was unsuccessful. Even after three generations, no significant movement toward $(R)$-stereoselectivity was observed. The most likely cause is that small side-chains need to be present simultaneously at two positions (78 and 113). Because only a single amino acid was replaced in each of the 13 first-generation libraries, it was impossible to identify a double mutant with better properties. This is the major drawback of the one-at-a-time iterative saturation mutagenesis strategy. The solution is to change two or positions simultaneously in a single library, as noted by Reetz. ${ }^{64}$ This must be balanced against the much greater number of possible variants. Pooling, reduced codon alphabets, and other methods have been devised to mitigate these issues. ${ }^{65}$

\section{Conclusions and Future Outlook}

In the 20 years since Massey's first report, enzyme-catalyzed alkene reductions have matured into a very useful addition to the synthetic toolkit. Wild-type enzymes often show very high stereoselectivities and a broad substrate tolerance. Their main deficiencies are a lack of naturally-occurring, stereocomplementary pairs and limited active site volumes. This stands in contrast to other biocatalyst classes, e.g., ketoreductases, transaminases and lipases.

As the studies summarized above have shown, protein engineering has been successfully employed to solve both major problems identified for alkene reductases. While changing one or a few amino acids can modestly increase the acceptable substrate size, the circular permutation approach of Lutz had by far the most dramatic improvement. Because circular permutation is a generally applicable technique that does not depend on detailed structural knowledge, this approach can be considered a first-line strategy for other alkene reductases. With regard to 
improving and / or inverting stereoselectivities, the best current strategy appears to be individual active site amino acid changes. Proximity to the bound substrate is a useful first-pass guide to choosing the targeted residues, although more beneficial impacts are often found by changing more distant amino acids. The combination of CASTing and ISM as developed by Reetz or a variation of these methods has proven quite useful in alkene reductases, and will likely remain the method of choice for the foreseeable future. Because these approaches depend on enzyme structural information, one must either determine a new alkene reductase structure experimentally by x-ray crystallography, or rely on computer models. One advantage of the CASTing / ISM strategy is that even an imperfect protein model is useful - the key information sought is the approximate distance between a residue and the bound substrate. Even if such estimates are incorrect by a few angstroms, it is unlikely to materially affect the choice of targeted residues.

One early concern was that every new substrate would demand a separate directed evolution campaign. In practice, however, variants selected against one substrate often show similar properties toward a range of substrates. ${ }^{56,66}$ If this observation proves general, it may be possible to maintain a reasonably small collection of alkene reductase pairs that provide both product enantiomers for a wide range of starting materials. This goal has already been partially achieved, and there is every reason to believe that further progress will be forthcoming, based on the lessons we have already learned.

\section{Acknolwedgements}

Our own work in this area was generously supported by the National Science Foundation (CHE-1111791). 


\section{Figure Legends}

Figure 1. Carvone binding to $S$. pastorianus OYE 1 in the "flipped" orientation. ${ }^{22}$ X-ray structures of wild-type OYE 1 and the Trp 116 Val (PDB code 4K8E) variant with bound $(S)$ carvone OYE 1 were overlaid. The solvent-accessible volume was calculated for the latter structure (shown as a semi-transparent surface). The isopropenyl group of $(S)$-carvone is wellaccommodated in this hydrophobic pocket. Entrance to this region is blocked by the indole sidechain of Trp 116 (red) in the wild-type enzyme, making it impossible for the substrate to bind in this manner to wild-type OYE 1.

Figure 2. Enzyme / substrate complex for cyclopentenone 5 and S. pastorianus Trp 116 Ile

OYE 1 (PDB code 3RND). ${ }^{23}$ Protein crystals were soaked with the ligand prior to data collection and two conformations of the ligand were observed (only the catalytically productive complex is shown with carbon atoms in light blue). The second, non-productive conformation is shown with carbon atoms in green. 


\section{References and Notes}

(1) Vaz, A. D. N.; Chakraborty, S.; Massey, V. Biochemistry 1995, 34, 4246-4256. Old Yellow Enzyme: Aromatization of Cyclic Enones and the Mechanism of a Novel Dismutation Reaction.

(2) Warburg, O.; Christian, W. Naturwissenschaften 1932, 20, 980-981. Über das Neue Oxydationsferment.

(3) Huang, M. M.; Hu, H. H.; Ma, L.; Zhou, Q.; Yu, L. S.; Zeng, S. Drug Metab. Rev. 2014, 46, 362-378. Carbon-Carbon Double-Bond Reductases in Nature.

(4) Toogood, H. S.; Scrutton, N. S. Curr. Opin. Chem. Biol. 2014, 19, 107-115. New Developments in 'Ene'-Reductase Catalysed Biological Hydrogenations.

(5) Hollmann, F.; Arends, I. W. C. E.; Holtmann, D. Green Chem. 2011, 13, 2285-2314. Enzymatic Reductions for the Chemist.

(6) Bougioukou, D. J. Ph.D., University of Florida, 2006.

(7) While this restriction would be lifted for large rings that can accommodate trans-alkenes, in practice, there has been little synthetic interest in such substrates.

(8) Originally known as Saccharomyces carlsbergensis.

(9) Fox, K. M.; Karplus, P. A. Structure 1994, 2, 1089-1105. Old Yellow Enzyme at 2 A Resolution: Overall Structure, Ligand Binding, and Comparison with Related Flavoproteins.

(10) Fox, K. M.; Karplus, P. A. J. Biol. Chem. 1999, 274, 9357-9362. The Flavin Environment in Old Yellow Enzyme - An Evaluation of Insights from Spectroscopic and Artificial Flavin Studies. 
(11) Haarer, B. K.; Amberg, D. C. Mol. Biol. Cell 2004, 15, 4522-4531. Old Yellow Enzyme Protects the Actin Cytoskeleton from Oxidative Stress.

(12) Haarer, B. K.; Amberg, D. C. Mol. Biol. Cell 2010, 21, 842. Old Yellow Enzyme Protects the Actin Cytoskeleton from Oxidative Stress (Retraction of Vol. 15, pg 4522, 2004).

(13) Karplus, P. A.; Fox, K. M.; Massey, V. FASEB J. 1995, 9, 1518-1526. Flavoprotein Structure and Mechanism. 8. Structure-Function Relations for Old Yellow Enzyme.

(14) Swiderska, M. A.; Stewart, J. D. J. Mol. Catal. B: Enzymatic 2006, 42, 52-54.

Stereoselective Enone Reductions by Saccharomyces carlsbergensis Old Yellow Enzyme.

(15) Brown, B. J.; Deng, Z.; Karplus, P. A.; Massey, V. J. Biol. Chem. 1998, 273, 32753-32762. On the Active Site of Old Yellow Enzyme. Role of Histidine 191 and Asparagine 194.

(16) Brown, B. J.; Hyun, J. W.; Duvvuri, S.; Karplus, P. A.; Massey, V. J. Biol. Chem. 2002, 277, 2138-2145. The Role of Glutamine 114 in Old Yellow Enzyme.

(17) Kohli, R. M.; Massey, V. J. Biol. Chem. 1998, 273, 32763-32770. The Oxidative HalfReaction of Old Yellow Enzyme - The role of Tyrosine 196.

(18) Meah, Y.; Massey, V. Proc. Natl. Acad. Sci. USA 2000, 97, 10733-10738. Old Yellow Enzyme: Stepwise Reduction of Nitro-Olefins and Catalysis of aci-Nitro Tautomerization.

(19) Xu, D.; Kohli, R. M.; Massey, V. Proc. Natl. Acad. Sci. USA 1999, 96, 3556-3561. The Role of Threonine 37 in Flavin Reactivity of the Old Yellow Enzyme.

(20) Straßner, J.; Fürholz, A.; Macheroux, P.; Amrhein, N.; Schaller, A. J. Biol. Chem. 1999, 274, 35067-35073. A Homolog of Old Yellow Enzyme in Tomato. Spectral Properties and Substrate Specificity of the Recombinant Protein. 
(21) Padhi, S. K.; Bougioukou, D. J.; Stewart, J. D. J. Am. Chem. Soc. 2009, 131, 3271-3280. Site Saturation Mutagenesis of Tryptophan 116 of Saccharomyces pastorianus Old Yellow Enzyme Uncovers Stereocomplementary Variants.

(22) Pompeu, Y. A.; B., S.; Stewart, J. D. ACS Catal. 2013, 3, 2376-2390. X-ray

Crystallography Reveals How Subtle Changes Control the Orientation of Substrate Binding in an Alkene Reductase.

(23) Walton, A. Z.; Conerly, W. C.; Pompeu, Y.; Sullivan, B.; Stewart, J. D. ACS Catalysis 2011, 1, 989-993. Biocatalytic Reductions of Baylis-Hillman Adducts.

(24) Abramovitz, A. S.; Massey, V. J. Biol. Chem. 1976, 251, 5321-5326. Purification of Intact Old Yellow Enzyme Using an Affinity Matrix for the Sole Chromatographic Step.

(25) A true Michaelis complex would involve alkene binding to OYE 1 whose FMN is in the reduced form.

(26) Daugherty, A. B.; Govindarajan, S.; Lutz, S. J. Am. Chem. Soc. 2013, 135, 14425-14432. Improved Biocatalysts from a Synthetic Circular Permutation Library of the FlavinDependent Oxidoreductase Old Yellow Enzyme.

(27) Positions within 6 amino acids of the original termini were left out of the library since they would be expected to differ little from the wild-type enzyme.

(28) Vick, B. A.; Zimmerman, D. C. Plant Physiol. 1986, 80, 202-205. Characterization of 12Oxo-phytodienoic Acid Reductase in Corn - The Jasmonic Acid Pathway.

(29) Schaller, F. J. Exp. Bot. 2001, 52, 11-23. Enzymes of the Biosynthesis of OctadecanoidDerived Signalling Molecules 
(30) Schaller, F.; Biesgen, C.; Mussig, C.; Altmann, T.; Weiler, E. W. Planta 2000, 210, 979984. 12-Oxophytodienoate Reductase 3 (OPR3) is the Isoenzyme Involved in Jasmonate Biosynthesis.

(31) Schaller, F.; Hennig, P.; Weiler, E. W. Plant Physiol. 1998, 118, 1345-1351. 12Oxophytodienoate-10,11-Reductase: Occurrence of Two Isoenzymes of Different Specificity Against Stereoisomers of 12-Oxophytodienoic Acid.

(32) Schaller, F.; Weiler, E. W. J. Biol. Chem. 1997, 272, 28066-28072. Molecular Cloning and Characterization of 12-Oxophytodienoate Reductase, an Enzyme of the Octadecanoid Signaling Pathway from Arabidopsis thaliana.

(33) Schaller, F.; Weiler, E. W. Eur. J. Biochem. 1997, 245, 294-299. Enzymes of Octadecanoid Biosynthesis in Plants - 12-Oxo-phytodienoate 10,11-reductase.

(34) Hall, M.; Stueckler, C.; Ehammer, H.; Pointner, E.; Oberdorfer, G.; Gruber, K.; Hauer, B.; Stuermer, R.; Kroutil, W.; Macheroux, P.; Faber, K. Adv. Synth. Catal. 2008, 350, 411-418. Asymmetric Bioreduction of $\mathrm{C}=\mathrm{C}$ Bonds Using Enoate Reductases OPR1, OPR3 and YqjM: Enzyme-Based Stereocontrol.

(35) Stueckler, C.; Hall, M.; Ehammer, H.; Pointner, E.; Kroutil, W.; Macheroux, P.; Faber, K. Org. Lett. 2007, 9, 5409-5411. Stereocomplementary Bioreduction of $\alpha, \beta$-Unsaturated Dicarboxylic Acids and Dimethyl Esters using Enoate Reductases: Enzyme- and Substrate-Based Stereocontrol.

(36) Winkler, C. K.; Clay, D.; Davies, S.; O'Neill, P.; McDaid, P.; Debarge, S.; Steflik, J.; Karmilowicz, M.; Wong, J. W.; Faber, K. J. Org. Chem. 2013, 78, 1525-1533. Chemoenzymatic Asymmetric Synthesis of Pregabalin Precursors via Asymmetric Bioreduction of $\beta$-Cyanoacrylate Esters Using Ene-Reductases. 
(37) Müller, A.; Hauer, B.; Rosche, B. Biotechnol. Bioeng. 2007, 98, 22-29. Asymmetric Alkene Reduction by Yeast Old Yellow Enzymes and by a Novel Zymomonas mobilis Reductase.

(38) Hall, M.; Stueckler, C.; Hauer, B.; Stuermer, R.; Friedrich, T.; Breuer, M.; Kroutil, W.; Faber, K. Eur. J. Org. Chem. 2008, 1511-1516. Asymmetric Bioreduction of Activated $\mathrm{C}=\mathrm{C}$ Bonds Using Zymomobilis mobilis NCR Enoate Reductase and Old Yellow Enzymes OYE 1-3 from Yeasts.

(39) Classen, T.; Pietruszka, J.; Schuback, S. M. ChemCatChem 2013, 5, 711-713. Revisiting the Enantioselective Sequence Patterns in Enoate Reductases.

(40) Oberdorfer, G.; Steinkellner, G.; Stueckler, C.; Faber, K.; Gruber, K. ChemCatChem 2011, 3, 1562-1566. Stereopreferences of Old Yellow Enzymes: Structure Correlations and Sequence Patterns in Enoate Reductases.

(41) Reich, S.; Kress, N.; Nestl, B. M.; Hauer, B. J. Struct. Biol. 2014, 185, 228-233. Variations in the Stability of NCR Ene Reductase by Rational Enzyme Loop Modulation.

(42) These choices were made by aligning a panel of both "classical" and "thermophilic" alkene reductases and comparing surface loop lengths.

(43) Binks, P. R.; E., F. C.; Nicklin, S.; Bruce, N. C. Appl. Environ. Microbiol. 1996, 62, 12141219. Degradation of Pentaerythritol Tetranitrate by Enterobacter cloacae PB2.

(44) Barna, T. M.; Khan, H.; Bruce, N. C.; Barsukov, I.; Scrutton, N. S.; Moody, P. C. F. J. Mol. Biol. 2001, 310, 433-447. Crystal Structure of Pentaerythritol Tetranitrate Reductase: "Flipped" Binding Geometries for Steroid Substrates in Different Redox States of the Enzyme. 
(45) Khan, H.; Barna, T.; Harris, R. J.; Bruce, N. C.; Barsukov, I.; Munro, A. w.; Mooday, P. C. E.; Scrutton, N. S. J. Biol. Chem. 2004, 279, 30563-30572. Atomic Resolution Structures and Solution Behavior of Enzyme-Substrate Complexes of Enterobacter cloacae PB2 Pentaerythritol Tetranitrate Reductase.

(46) Fryszkowska, A.; Toogood, H.; Sakuma, M.; Gardiner, J. M.; Stephens, G. M.; Scrutton, N. S. Adv. Synth. Catal. 2009, 351, 2976-2990. Asymmetric Reduction of Activated Alkenes by Pentaerythritol Tetranitrate Reductase: Specificity and Control of Stereochemical Outcome by Reaction Optimisation.

(47) Toogood, H. S.; Fryszkowska, A.; Hare, V.; Fisher, K.; Roujeinikova, A.; Leys, D.; Gardiner, J. M.; Stephens, G. M.; Scrutton, N. S. Adv. Synth. Catal. 2008, 350, 2789-2803. Structure-Based Insight into the Asymmetric Bioreduction of the $\mathrm{C}=\mathrm{C}$ Double Bond of $\alpha, \mathrm{b}$ Unsaturated Nitroalkenes by Pentaerythritol Tetranitrate Reductase.

(48) Fryszkowska, A.; Toogood, H. S.; Mansell, D.; Stephens, G.; Gardiner, J. M.; Scrutton , N. S. FEBS J. 2012, 279, 4160-4171. A Surprising Observation that Oxygen Can Affect the Product Enantiopurity of an Enzyme-Catalyzed Reaction.

(49) Hulley, M.; Toogood, H. S.; Fryszkowska, A.; Mansell, D.; Stephens, G. M.; Gardiner, J. M.; Scrutton, N. S. ChemBioChem 2010, 11, 2433-2447. Focused Directed Evolution of Pentaerythritol Tetranitrate Reductase by Using Automated Anaerobic Kinetic Screening of Site-Saturated Libraries.

(50) Oxygen was excluded, both because it can act as a competing electron acceptor and also because it was shown to influence the stereoselectivity of PETN reductions (Ref. X).

(51) No significant changes were apparent when the native and His-tagged PETN reductase structures were compared. 
(52) Fryszkowska, A.; Toogood, H.; Sakuma, M.; Stephens, G. M.; Gardiner, J. M.; Scrutton, N. S. Catal. Sci. Technol. 2011, 1, 948-957. Active Site Modification in Pentaerythritol Tetranitrate Reductase Can Lead to Improved Product Enantiopurity, Decreased ByProduct Formation and Altered Stereochemical Outcome in Reactions with $\alpha, \beta-$ Unsaturated Nitroolefins.

(53) Toogood, H.; Fryszkowska, A.; Hulley, M.; Sakuma, M.; Mansell, D.; Stephens, G. M.; Gardiner, J. M.; Scrutton, N. S. ChemBioChem 2011, 12, 738-749. A Site-Saturation Mutagenesis Study of Pentaerythritol Tetranitrate Reductase Reveals that Residues 181 and 184 Influence Ligand Binding, Stereochemistry and Reactivity.

(54) Reetz, M. T. Angew. Chem. Int. Ed. 2011, 50, 138-174. Laboratory Evolution of Stereoselective Enzymes: A Prolific Source of Catalysts for Asymmetric Reactions.

(55) Reetz, M. T.; Krebs, G. P. L. Comptes Rendus Chimie 2011, 14, 811-818. Challenges in the Directed Evolution of Stereoselective Enzymes for Use in Organic Chemistry.

(56) Bougioukou, D. J.; Kille, S.; Taglieber, A.; Reetz, M. T. Adv. Synth. Catal. 2009, 351, 3287-3305. Directed Evolution of an Enantioselective Enoate-Reductase: Testing the Utility of Iterative Saturation Mutagenesis.

(57) Walton, A. Z.; Sullivan, B.; Patterson-Orazem, A. ACS Catalysis 2014, submitted for publication. Residues Controlling Facial Selectivity in an Alkene Reductase and SemiRational Alterations to Create Stereocomplementary Variants.

(58) Jeffries, T. W.; Grigoriev, I. V.; Grimwood, J.; Laplaza, J. M.; Aerts, A.; Salamov, A.; Schmutz, J.; Lindquist, E.; Dehal, P.; H., S.; Jin, Y.-S.; Passoth, V.; Richardson, P. M. Nature Biotech. 2007, 25, 319-326. Genome Sequence of the LignocelluloseBioconverting and Xylose-Fermenting Yeast Pichia stipitis. 
(59) Trp 116 in S. pastorianus OYE 1.

(60) Bougioukou, D. J.; Walton, A. Z.; Stewart, J. D. Chem. Commun. 2010, 46, 8558-8560. Toward Preparative-Scale, Biocatalytic Alkene Reductions.

(61) Pompeu, Y. A.; Sullivan, B.; Walton, A. Z.; Stewart, J. D. Adv. Synth. Catal. 2012, 354, 1949-1960. Structural and Catalytic Characterization of Pichia stipitis OYE 2.6, a Useful Biocatalyst for Asymmetric Alkene Reductions.

(62) Sullivan, B.; Walton, A. Z.; Stewart, J. D. Enzyme Microb. Technol. 2013, 53, 70-77. Library Construction and Evaluation for Site Saturation Mutagenesis.

(63) The "flipped" binding mode predicts $(R)$-stereoselectivity, consistent with the experimental observation.

(64) Reetz, M. T.; Carballeira, J. D. Nature Prot. 2007, 2, 891-903. Iterative Saturation Mutagenesis (ISM) for Rapid Directed Evolution of Functional Enzymes.

(65) Reetz, M. T. J. Am. Chem. Soc. 2013, 135, 12480-12496. Biocatalysis in Organic Chemistry and Biotechnology: Past, Present, and Future. 


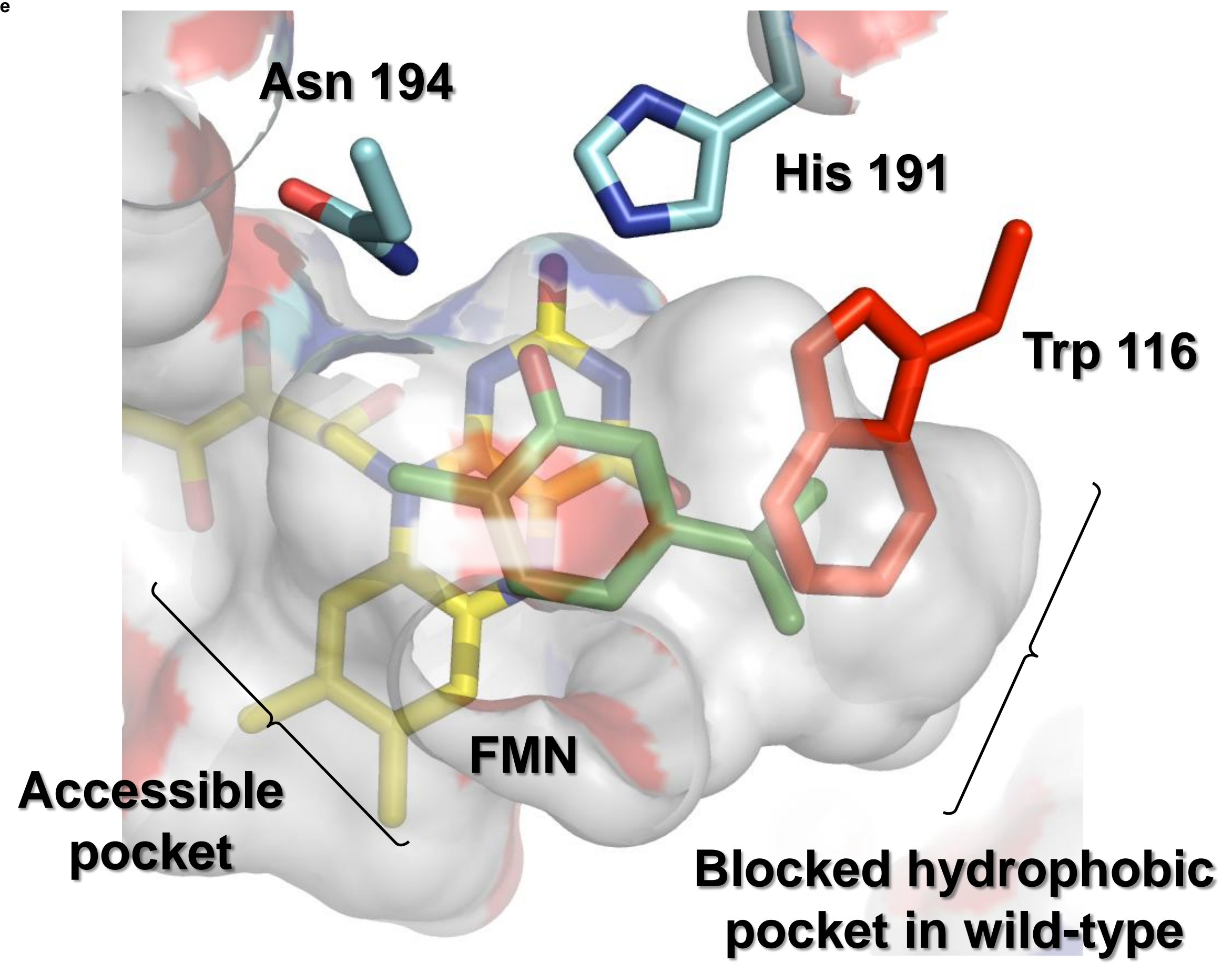

\section{Blocked hydrophobic pocket in wild-type}




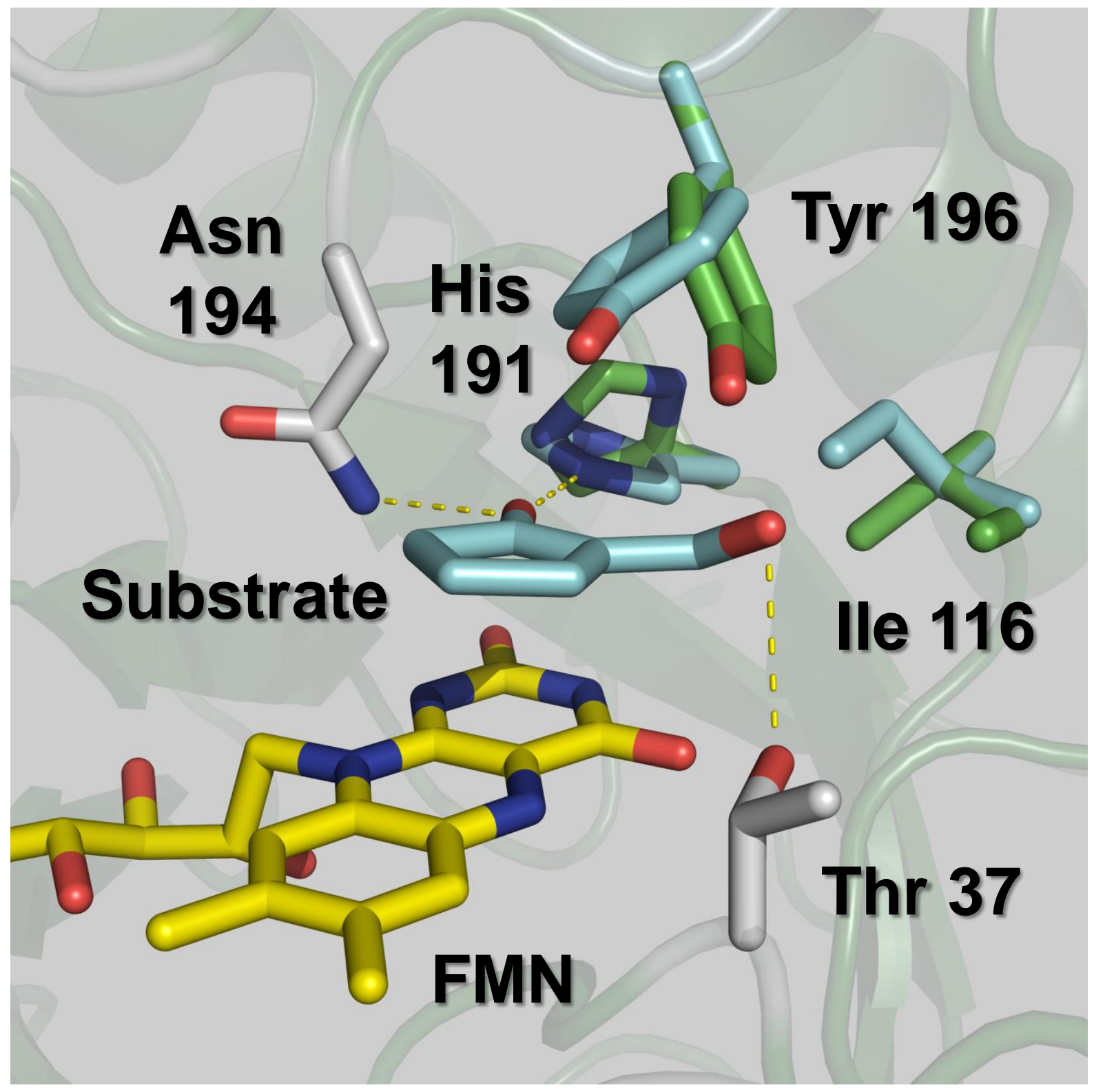




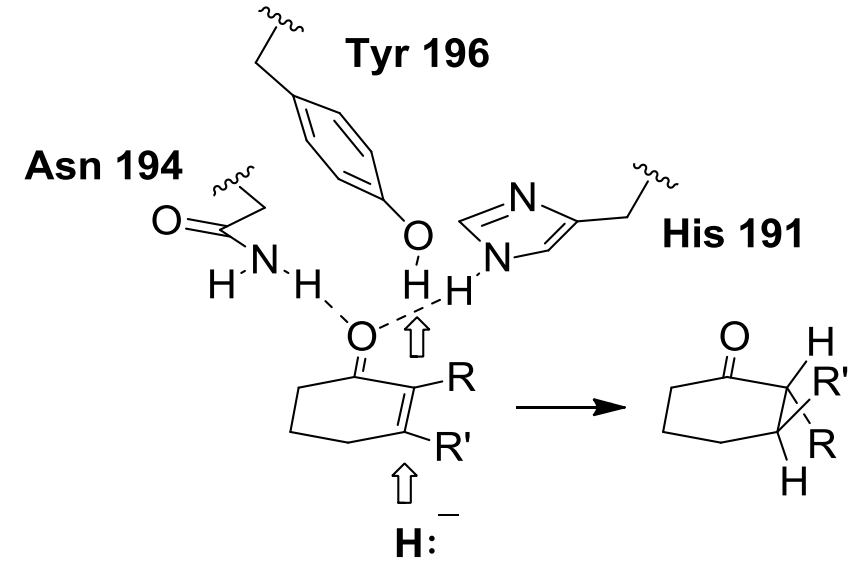

Scheme 1 (from $\mathrm{FMNH}_{2}$ ) 


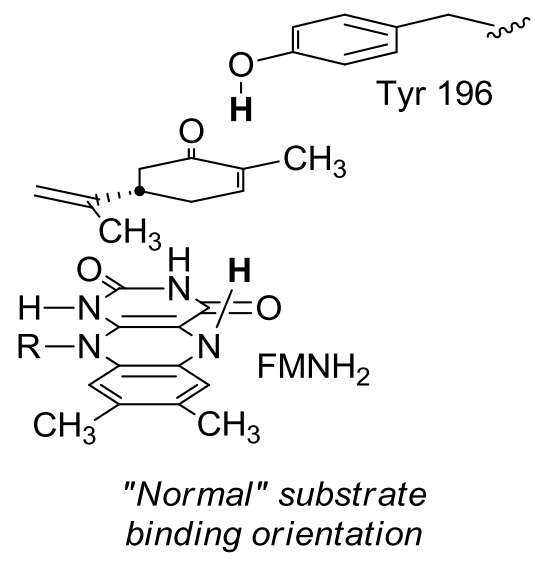

Scheme 2

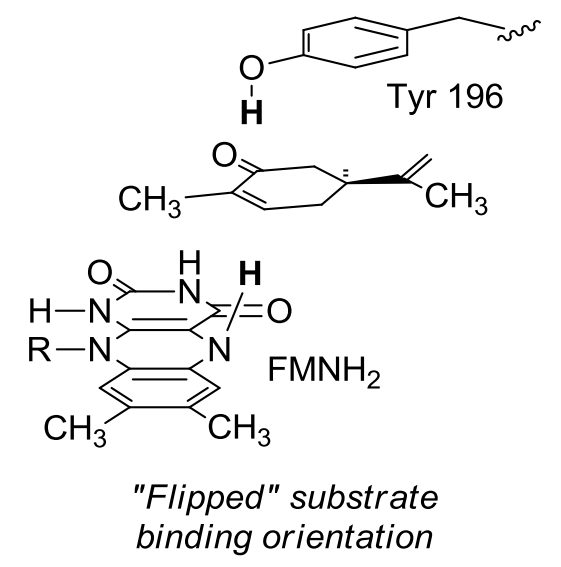

binding orientation 

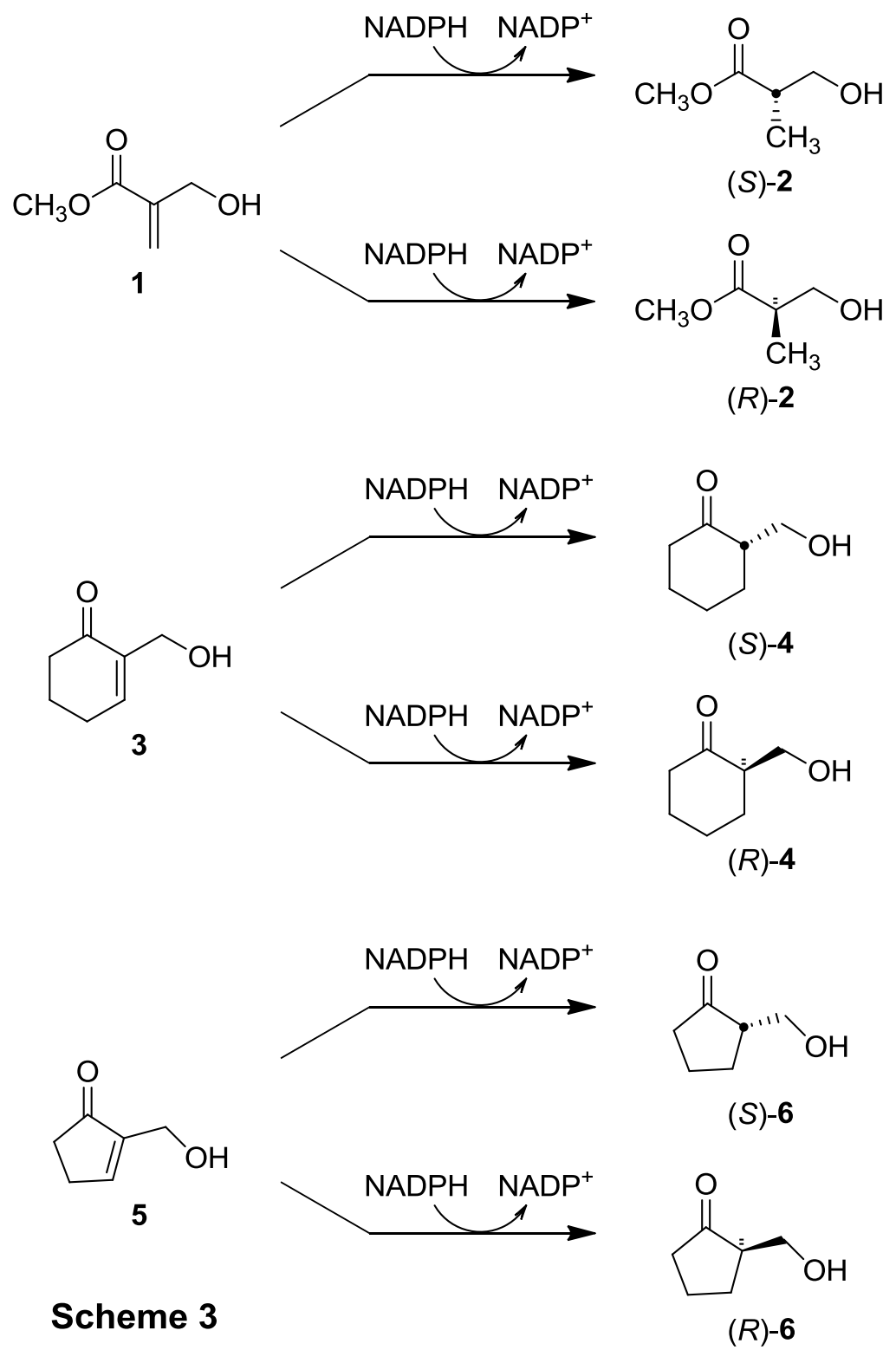


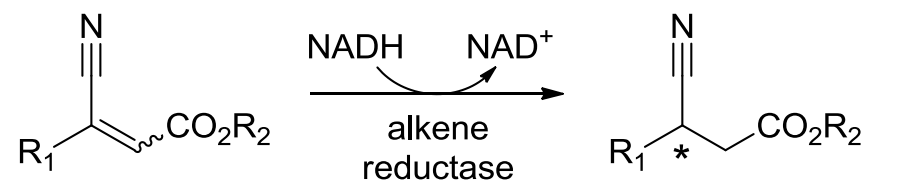

$\mathrm{R}_{1}=\mathrm{Me}, \mathrm{Et}, n-\mathrm{Pr}, i-\mathrm{Bu}$

$\mathrm{R}_{2}=\mathrm{Me}, \mathrm{Et}, n-\mathrm{Pr}$

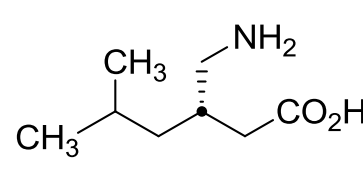

(S)-Pregabalin

\section{Scheme 4}

